\title{
Sistema de ventas para librería Sánchez, Siuna, 2016
}

Sales System for Sánchez Bookstore, Siuna, 2016

\section{Walter Garzón Díaz' \\ Griselda Reyes Macís ${ }^{2}$ \\ Frank Robing Salazar Solano ${ }^{3}$}

\section{Resumen}

耳 ste estudio se realizó en librería Sánchez, ubicada en el barrio "Pedro Joaquín Chamorro" sector 3 de Ela ciudad de Siuna-RACCN, Nicaragua. El objetivo fue desarrollar un sistema de control de ventas e inventario; para esto se realizó una base de datos, para el registro de entradas y salidas de los productos del negocio, además se diseñó una interfaz que permite al usuario interactuar con la información almacenada en la base de datos y posteriormente la implementación del sistema. El estudio fue aplicativo desde el paradigma cuantitativo.

Se desarrolló el sistema y se implementó en la librería Sánchez. El sistema permite a los usuarios, ingresar datos de todos los productos y sus categorías, clientes, proveedores existentes, además facilita las gestiones de ventas y compras.

Palabras Clave: control de ventas; inventario; librería; Sistema.

\section{Summary}

This study was conducted in Sanchez Bookstore, located in the "Pedro Joaquín Chamorro" neighborhood, sector 3 of the city of Siuna-RACCN, Nicaragua. The objective was to develop a sales and inventory control system; For this, a database was made for the registration of inputs and outputs of the business products, in addition an interface was designed that allows the user to interact with the information stored in the database and later the implementation of the system. The study was applied from the quantitative paradigm.

The system was developed and implemented in the Sanchez bookstore. The system allows users to enter data on all products and their categories, customers, existing suppliers, and also facilitates sales and purchases.

Key Words: Sales Control; Inventory; Bookstore; System

\footnotetext{
1 Licenciado en Informática Administrativa por la Universidad de las Regiones Autónomas de la Costa Caribe Nicaragüense-Recinto Universitario Las Minas. Correo:gwalasd@gmail.com

2 Licenciada en Informática Administrativa por la Universidad de las Regiones Autónomas de la Costa Caribe Nicaragüense-Recinto Universitario Las Minas. Correo: reyesmacisgris@gmail.com

3 Licenciado en Informática Administrativa. Profesor de la Universidad de las Regiones Autónomas de la Costa Caribe Nicaragüense-Recinto Universitario Las Minas. Correo: sfrankrobinson@gmail.com
} 


\section{Introducción}

El término librería es utilizado para designar a aquellos establecimientos y espacios en los que el principal producto en venta son los libros, aunque los mismos pueden estar complementados por revistas, diarios, útiles escolares, y por otros materiales, como sucede en la actualidad.

La librería más antigua del mundo está en Lisboa, La librería Bertrand fue fundada en 1732, así que lleva ya más de dos siglos y medios de historia. (Domínguez, 2014).

Durante los últimos años los sistemas de información constituyen uno de los principales ámbitos de estudio en el área de organización de empresas. El entorno donde las compañías desarrollan sus actividades se vuelve cada vez más complejo.

Jahn (2013), asegura que en Nicaragua hoy día cualquier empresa formal tiene su sistema de facturación. Además destaca que tiene gran importancia $y$ hay un avance en Nicaragua.

La librería Sánchez, cuenta con una variedad de productos; sin embargo, el control de inventarios se realiza de forma manual utilizando cuadernos tradicionales. Esto ocasionaba que el proceso sea más lento para controlar las entradas y salidas de los productos.

Mediante esta investigación se desarrolló un sistema para el control del registro mediante la creación de una base de dato y una interfaz de usuario.

El beneficio de desarrollo de este sistema es que certifique una mejor atención más rápida y eficiente para agilizar los procesos de inventario y lograr un mejor control de los productos que ingresan y salen en la librería Sánchez. Este sistema cubre las necesidades y brinda un mejor control de las entradas y salidas de los productos, permitiendo una mayor efectividad en el manejo del flujo y procesamientos de los datos.

La implementación de este sistema tiene mucha importancia para la librería Sánchez, el cual permitirá a la misma, una información confiable, agilizando y facilitando la realización de las tareas efectuadas por el negocio.

\section{Revisión de literatura}


pectos técnicos, se emplea el término interfaz para definir el juego de conexiones y dispositivos que hacen posible la comunicación entre dos sistemas.

Cuando se habla de interfaz se hace referencia a la cara visible de los programas tal y como se presenta a los usuarios para que interactúen con la máquina. La interfaz gráfica implica la presencia de un monitor de ordenador o pantalla constituida por una serie de menús e iconos que representan las opciones que el usuario puede tomar dentro del sistema.

Ríos y Moran (2014), exponen que el diseño de la interfaz de usuario es el mecanismo por el cual el usuario interactúa con la herramienta, equipo, dispositivo o aplicación específica permitiendo un óptimo funcionamiento del sistema.

\section{Implementación de sistemas de información}

De acuerdo a Kendall y Kendall, la implementación es la última fase del desarrollo de sistemas, el analista ayuda a implementar el sistema de información. En esta fase hay que capacitar a los usuarios para operar el sistema (2011, p. 11).

Según Fernández (2012), dentro del ciclo de vida se encuentra la fase de implementación de un sistema, es la fase más costosa y que consume más tiempo, se dice que es costosa porque muchas personas, herramientas y recursos, están involucrados en el proceso y consume mucho tiempo porque se completa todo el trabajo realizado previamente durante el ciclo de vida.

En la fase de implementación se instala el nuevo sistema de información para que empiece a trabajar y se capacita a sus usuarios para que puedan utilizarlo. La instalación puede realizarse según cuatro métodos: Directo, paralelo, piloto y en fases (Fernández, 2012).

Método directo: Se abandona el sistema antiguo y se adopta inmediatamente el nuevo.

Método paralelo: Los sistemas de información antiguo y nuevo operan juntos hasta que el nuevo demuestra ser confiable.

Método piloto: Pone a prueba el nuevo sistema sólo en una parte de la organización. Método en fases: La implementación del sistema se divide en partes o fases, que se van realizando a lo largo de un periodo de tiempo, sucesivamente.

Los métodos piloto y en fases suelen ser los más practicados puesto que tienen menor riesgo. Como se puede observar la decisión de adoptar cualquiera de los métodos estará influenciada por factores de riesgo y disponibilidad de recursos (Fernández, 2012).

Otro aspecto importante de esta fase es la capacitación del personal, que cobra especial importancia para asegurar el uso acertado del sistema. Se puede adelantar camino al capacitar personal, antes incluso de contar con los equipos nuevos, para que el usuario se familiarice con el nuevo sistema. Si el sistema es sencillo y el usuario tiene cierta experiencia, la capacitación formal no se hace necesaria y bastarán algunas instrucciones para ponerle al tanto (Fernández, 2012).

\section{Materiales y métodos}

El estudio se realizó en la librería Sánchez, ubicada en el barrio Pedro Joaquín Chamorro sector 3 de la ciudad de Siuna RACCN, Nicaragua, es aplicativo bajo el paradigma cuantitativo; el universo está compuesto por todas las librerías del municipio de Siuna, la unidad de análisis está compuesta por propietario y trabajadores de la librería, las fuentes primarias de información es el área de ventas (propietario y trabajadores) a los cuales se les aplicaron entrevistas, los datos obtenidos fueron procesados en el editor de textos Microsoft Word y en la hoja de cálculos Microsoft Excel.

\section{Resultados y discusión}

Los resultados del estudio investigativo en la creación del sistema de ventas de la librería Sánchez, ubicada en el barrio Pedro Joaquín Chamorro sector 3; están presentados de forma clara y ordenada de acuerdo a los objetivos planteados. 


\section{Base de datos en Structured Query Language SQL Server 2008}

Sobre la importancia de las bases de datos Estrada (2015) relata que, facilitan no solo el registro de gran cantidad de datos, sino también el acceso de los mismos; lo cual permite ahorrar espacio físico como tiempo para consultar información de ella.

Coincidiendo con lo dicho por Estrada, las bases de datos cumplen el papel de garantizar a un usuario la facilidad en el almacenamiento y procesamiento de datos e información.

Microsoft (s.f.), afirma que Structured Query Language SQL Server 2008 R2, es un sistema de administración de datos eficaz y confiable que ofrece un variado conjunto de características, protección de datos y rendimiento para aplicaciones, sitios web ligeros y almacenes de datos locales.

Para crear la base de datos, se instaló el gestor de bases de datos de Structured Query Language SQL Server 2008 R2, que permite administrar bases de datos de manera eficaz y confiable como lo describe Microsoft.

Una vez finalizada la instalación del gestor Structured Query Language SQL Server, se creó la base de datos Librería, la cual contiene 11 (once) tablas necesarias para el correcto almacenamiento de los datos (ver figura 2).

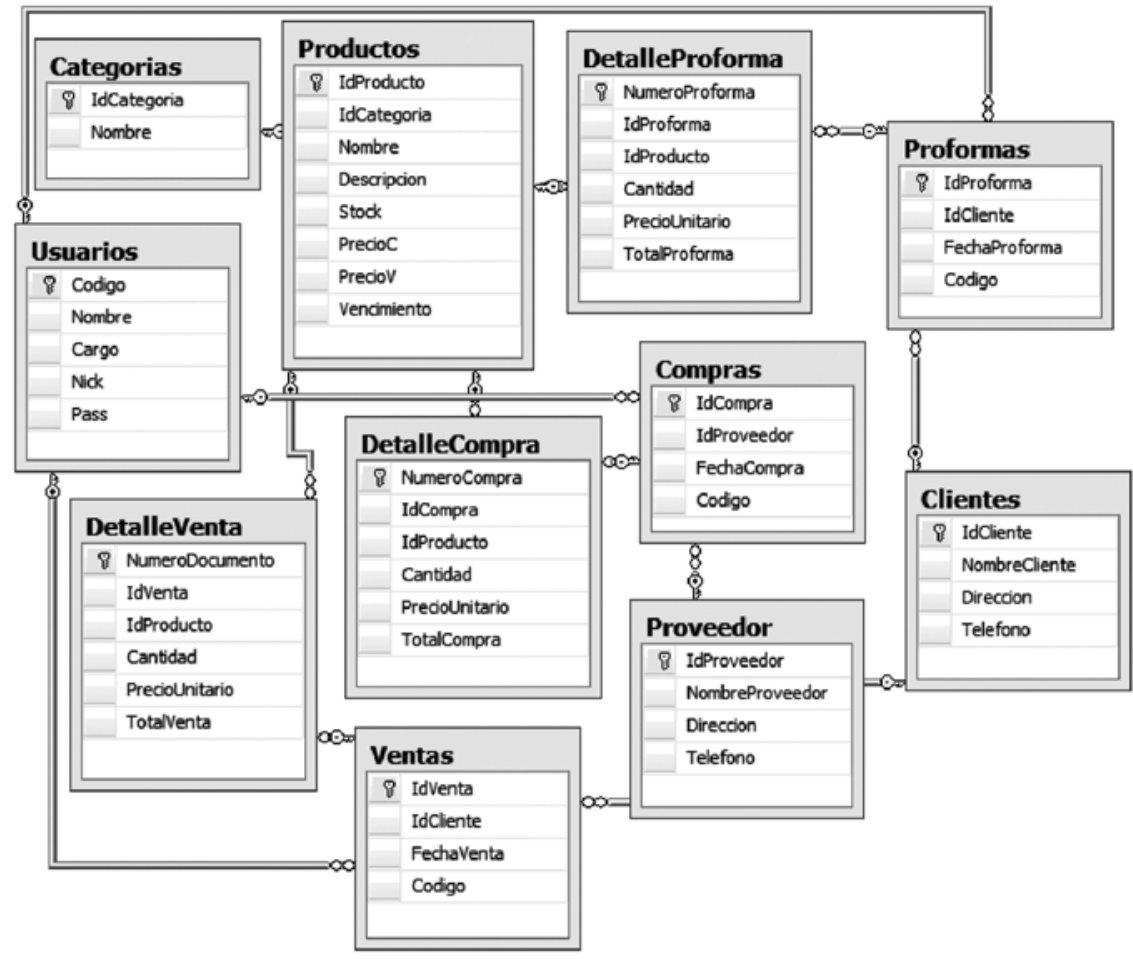

Figura 2: Diagrama de la base de datos Librería

La base de datos Librería, confirma lo dicho por Masadelante (s.f.), quién afirma: que una base de datos es una colección de información organizada de forma que un programa de ordenador pueda seleccionar rápidamente los fragmentos de datos que necesite. Una base de datos es un sistema de archivos electrónico.

El proceso de normalización se realizó aplicando la primera, segunda y tercera forma normal ( $1 \mathrm{FN}, 2 \mathrm{FN}$ y ${ }_{3} \mathrm{FN}$ ) coincidiendo con lo que dice EcuRed (s.f.), quién afirma que "Las primeras tres formas normales son suficientes para cubrir las necesidades de la mayoría de las bases de datos". 
Después de diseñar la base de datos, se crearon 82 procedimientos almacenados necesarios para realizar todas las consultas desde una interfaz gráfica de usuario.

Según la Ley de concertación tributaria (2012), para la determinación del costo de los bienes y servicios producidos, el contribuyente podrá escoger cualquiera de estos métodos siguientes: costo promedio, primera entrada, primera salida y última entrada, primera salida.

Coincidiendo con lo descrito en la Ley de concertación tributaria, para la valuación de inventarios se crearon procedimientos almacenados de acuerdo al método UEPS (Últimos en Entrar Primeros en Salir), ya que los precios de los productos están en constante ascenso.

De acuerdo a Salazar (2016), UEPS es un método de valoración basado en que los últimos artículos que entraron a formar parte del inventario, son los primeros en venderse, claro está en función del costo unitario, es decir que el flujo físico es irrelevante, aquí lo importante es que el costo unitario de las últimas entradas sea el que se aplique a las primeras salidas.

\section{Interfaz gráfica en Visual Studio 2012}

Ríos y Moran (2014), exponen que el diseño de la interfaz de usuario es el mecanismo por el cual el usuario interactúa con la herramienta, equipo, dispositivo o aplicación específica permitiendo un óptimo funcionamiento del sistema.

Constatando lo dicho por Ríos y Moran, la interfaz gráfica de sistema Ls System fue diseñada en el lenguaje de programación Visual Basic .Net 2012, ya que es una herramienta de que permite desarrollar aplicaciones profesionales.

Ls System se diseñó bajo el paradigma orientado a objetos ya es una forma de programación imperativa que describe la secuencia que debe seguir el programa para resolver un problema dado, esta definición concuerda con lo descrito por 4 rSoluciones (2013), quien afirma que en el paradigma orientado a objetos, el comportamiento del programa es llevado a cabo por objetos, entidades que representan elementos del problema a resolver y tienen atributos y comportamiento.

La interfaz gráfica se diseñó coincidiendo con lo dicho por Lamarca (2013), quien afirma que una de las principales características de la interfaz gráfica debe basarse en un diseño ergonómico mediante el establecimiento de menús, barras de acciones e iconos de fácil acceso.

Ls System contiene 42 formularios cada uno con sus respectivos botones y controles codificados para que el usuario interactúe con el sistema.

Cada ventana (formulario) posee controles dependiendo de la función que cumple.

La pantalla o menú principal del sistema tiene los siguientes accesos:

1) Barra de menú: contiene los accesos a casa tabla o formulario del sistema.

2) Barra de acceso rápido (mediante esta opción se puede acceder de forma directa a: Cerrar sesión, Reiniciar, Salir, Usuarios, Ventas, Proformas y Compras).

3) Botones minimizar y cerrar.

4) Nombre del sistema.

5) Hora y fecha.

6) Centro o área de trabajo (ver grafica 3). 


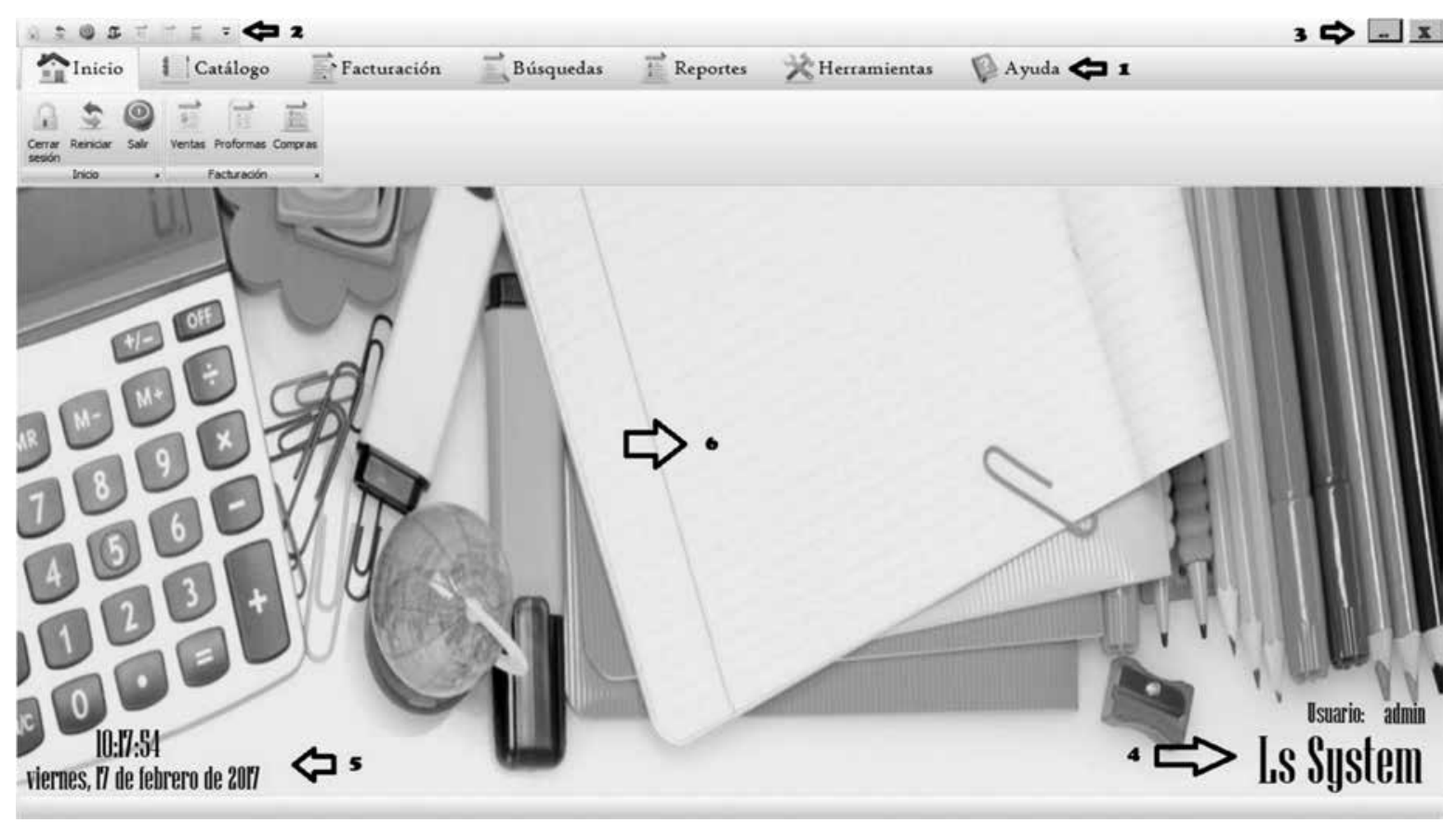

Gráfica 3. Pantalla principal del sistema Ls System

\section{Implementación del sistema}

\section{a) Instalador de Ls System}

De acuerdo a Kendall y Kendall, la implementación es la última fase del desarrollo de sistemas, el analista ayuda a implementar el sistema de información. En esta fase hay que capacitar a los usuarios para operar el sistema (2011, p. 11).

Después de finalizado el diseño del sistema y de acuerdo a Kendall y Kendall, se procedió a crear el instalador del software utilizando el asistente para la creación de aplicaciones de visual Studio 2012 y posteriormente a realizar la instalación del sistema.

\section{b) Instalación de Ls System}

El sistema Ls System se instaló en un equipo de cómputo de la librería Sánchez destinado para tal función, bajo las siguientes especificaciones:

Sistema operativo Windows 7 ultimate.

.Net Framework 4.5.

Memoria RAM: 2 GB

Espacio en disco duro: 6o GB. 


\section{c) Capacitación a usuarios}

En el proceso de capacitación de los usuarios finales, se les explicó detalladamente cada función del sistema siguiendo paso a paso las instrucciones descritas en el manual de usuario (ver anexo 6) y coincidiendo con lo expuesto por SYSTEMNOR (2012), quien afirma que se debe capacitar tanto a los usuarios que vayan a interactuar en el sistema directamente como los que indirectamente se vean afectados para que comprendan el proceso que lleva al registrar, dar servicio técnico, recibir, clasificar y/o escalar una incidencia.

\section{d)Pruebas del sistema Ls System}

Según González (2013), en las pruebas alfa y beta el código fuente no está probado, y la atención se centra en la funcionalidad del software, de acuerdo con las necesidades del usuario. Pruebas Alfa: se llevan a cabo, por un cliente, en el lugar de desarrollo. Se usa el software de forma natural con el desarrollador como observador del usuario, registrando los errores y problemas de uso. Prueba Beta: se llevan a cabo por los usuarios finales del software en los lugares de trabajo de los clientes. A diferencia de la prueba alfa, el desarrollador no está presente normalmente.

Coincidiendo con lo dicho por González, las pruebas del sistema se realizaron de la siguiente manera:

Alfa: el sistema se instaló en un equipo de cómputo y se sometió a prueba bajo la supervisión de los programadores.

Beta: se instaló el software en el equipo designado por el propietario de librería Sánchez con un periodo de prueba de 30 días. Dando como resultado el correcto funcionamiento del mismo (ver gráfica 4).

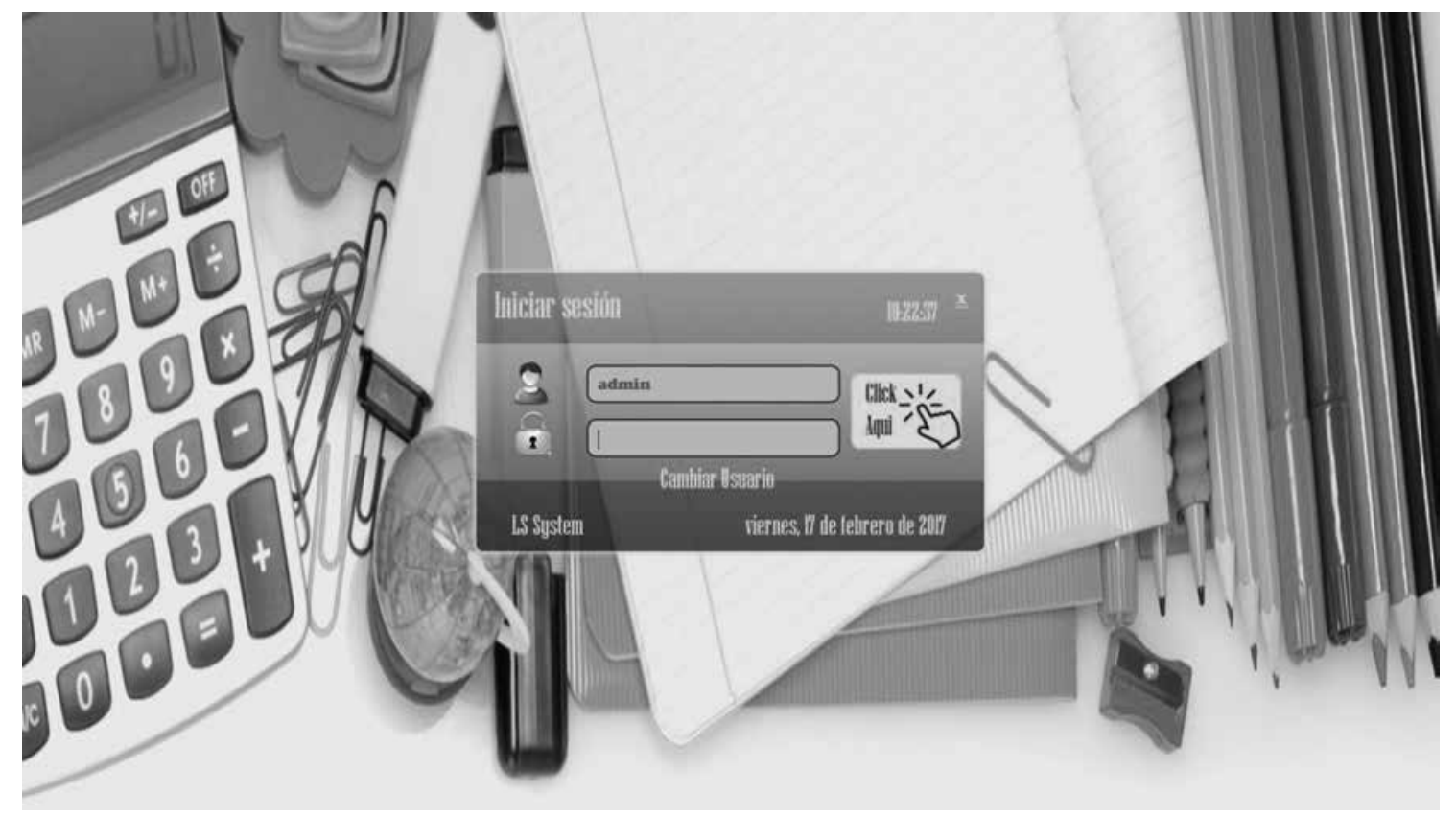

Figura 4. Inicio de sesión del sistema Ls System. 


\section{e) Manual de usuario}

El manual de usuario del sistema Ls System, se le entregó de manera impresa al propietario de la librería Sánchez. Además, se incorporó en el menú de ayuda del software.

\section{Conclusiones}

El estudio realizado dio como resultado el desarrollo de un sistema de información, al que se denominó LS System.

Utilizando Microsoft Structured Query Language SQL server 2008, se creó una base de dato denominada Librería, la cual está conformada por 11 tablas, debidamente normalizadas y relacionadas entre sí, mediante claves primarias y secundarias, evitando de esta manera la redundancia e inconsistencia de datos.

La interfaz de usuario fue diseñada mediante el lenguaje de programación visual Basic.NET 2012. Para esto se diseñó un total de 42 formularios que le permitan al usuario interactuar con la base de datos de una manera fácil, sencilla y segura obteniendo información precisa y en tiempo real.

Mediante el asistente de publicación de visual Basic, se creó un ejecutable del sistema; el cual se utilizó para realizar la implementación o instalación del mismo en un equipo de computadora de la librería Sánchez. A los usuarios finales se les brindo una capacitación sobre el uso adecuado del software.

\section{Recomendaciones}

\section{Al propietario de Librería Sánchez:}

- Dar mantenimiento correctivo y preventivo al equipo donde se encuentre instalado el sistema.

- Determinar la seguridad del equipo donde está instalado el sistema, en cuanto al ambiente que este se encuentre ubicado.
- Realizar respaldo periódicamente para mantener una copia de seguridad actualizada de la base de dato.

\section{A los usuarios del sistema Ls System:}

- Utilizar el manual de usuario como método de ayuda rápida y preventiva en la manipulación del sistema.

- Evitar instalar programas no apropiados en la computadora para evitar algún problema de software.

\section{Lista de referencias}

4rSoluciones. (2013). ¿Qué son los paradigmas de programación? Recuperado de http://www.4rsoluciones.com/blog/ que-son-los-paradigmas-de-programacion-2/

Domínguez, C. (2014). LA LIBRERÍA MÁS ANTIGUA DEL MUNDO ESTÁ EN LISBOA. Recuperado de: http://www.libropatas. com/libros-literatura/la-libreria-masantigua-del-mundo-esta-en-lisboa/

EcuRed. (s.f.). Normalización d e una base de datos. Recuperadoel tps://www.ecured.cu/Normalizaci\%C3\%B3n_ de_una_base_de_datos

Estrada, R. (2015). La importancia de las bases de datos en la actualidad. Recuperado de https://prezi.com/wmtnk8zil6qp/la-importancia-de-las-bases-de-datos-en-laactualidad/

Fernandez, N. (2012). Fase de implementación de sistemas de información. Recuperado de http://es.slideshare.net/NAHAMA19/ fase-de-implementacin-desistemas-de-informacin

Gonzalez, J. (2013). Pruebas Alfa y beta. Recuperado de http://pruebasalfaybeta.blogspot.com/

Instituto de Nutrición de Centro América y Panamá. (s.f.). Sistema de Información. Recuperado de http://www.incap.int/sisvan/index.php/es/acerca-desan/conceptos/ sistema-de-vigilancia 
Iruela, J. (2016). Los gestores de bases de datos más usados. Recuperado de http:// revistadigital.inesem.es/informatica-y-tics/ los-gestores-de-bases-dedatos-mas-usados/

Jahn, H. (2013). “Las tecnologías de la información aumentan la productividad". Recuperado de http://www.elnuevodiario.com.ni/economia/275858-tecnologiasinformacion-aumentan-productividad/

Kendall, K. \& Kendall, J. (2011). ANÁLISIS Y DISEÑO DE SISTEMAS (8va ed.).

México: PEARSON EDUCACIÓN

Lamarca, M. (2013). La interfaz gráfica. Recuperado de http://www.hipertexto.info/documentos/ interfaz.htm

Ley No. 822. Ley de concertación tributaria. Publicada en La Gaceta Diario Oficial No.

241, del 17 de diciembre de 2012. Nicaragua.

Masadelante.com. (s.f.). ¿Qué es una Base de datos? Definición de Base de datos. Recuperado de http://www.masadelante.com/faqs/ base-de-datos

Mestra, M. (2012). SAV (SISTEMA AUTOMATIZADO DE VENTAS). Recuperado de: http://elmejorblogsdeatecionalcliente.blogspot. $\mathrm{com} / 2012 / 10 / \mathrm{sav}$-sistemaautomatizado-de-ventas.html

Microsoft. (s.f.). Microsoft SQL Server 2008 R2 RTM - Management Studio Express.

Recuperado de https://www.microsoft.com/ eses/download/details.aspx?id=22985

Ríos, A., Moran, V., (2014). Diseño de Interfaz De usuario-Ingeniería del software. Recuperado de: https://prezi.com/nrqrqnrzps_v/diseno-de-interfaz-de-usuarioingenieria-del-software/

Salazar, B. (2016). MÉTODOS DE VALORACIÓN DE INVENTARIOS. Recuperado de https:// www.ingenieriaindustrialonline.com/herramientas-para-el-ingenieroindustrial/administraci\% $\mathrm{C}_{3} \% \mathrm{~B}_{3}$ n-de-inventarios $/ \mathrm{m} \% \mathrm{C}_{3} \% \mathrm{~A}$ 9todos-devaloraci\% $\mathrm{C}_{3} \% \mathrm{~B}_{3}$-de-inventarios/

Sánchez, L., Payano, R., \& Bonilla, J. (2014). Base de datos. Recuperado de http://es.slideshare. net/lujusanch/base-de-datos-14086052

SYSTEMNOR. (2012). ESTRATEGIA DE CAPACITACION PARA LA IMPLANTACION DE UN NUEVO SISTEMA DE INFORMACION (SOFTWARE). Recuperado de https:// systemnor.wordpress.com/2012/06/29/ estrategia-de-capacitacion-parala-implantacion-de-un-nuevo-sistema-de-informacion-software/ 